\title{
Research 2021: never the same again
}

\section{DOI: 10.7861/fhj.ed.8.2.2}

This issue of the Future Healthcare Journal (FHJ) focuses on some of the future research considerations as we emerge from the COVID-19 pandemic. In a collection of excellent contributions jointly commissioned by the Royal College of Physicians (RCP) and The Academy of Medical Sciences (AMS), we will see how the sector pushes for the best research to benefit patients and the public. As the world seeks to learn lessons from the COVID-19 pandemic, the exemplary research from the UK makes this $\mathrm{FH}$ ] issue of timely importance.

For too long, research has been viewed and increasingly operationalised as being separate from clinical care despite being a key principle of the NHS. ${ }^{1}$ Often viewed as an 'activity if time and cost permit', research has increasingly had a narrowing geographical focus rather than where clinical need is greatest, thereby widening health inequalities. As this issue will highlight, COVID-19 has been an exemplar of how an integrated research ecosystem has come together across all of the UK and that the dial for research needs to be reset at the heart of clinical care.

A promising development is the UK government publication in March 2021, Saving and improving lives: the future of UK clinical research delivery. ${ }^{2}$ It echoes many of the policy calls from the RCP and AMS, especially around the need to embed research in the NHS, the importance of patient-centred research and the need to have a sustainable and well supported workforce. ${ }^{3,4}$

Another timely and important driver is the recently published Care Quality Commission (CQC) strategy for 2021, A new strategy for the changing world of health and social care. ${ }^{5}$ It builds upon earlier recognition of research activity within the well-led domain in CQC inspections and commits to services playing an active part in research to improve care and creating a culture where innovation and research can flourish.

These are important steps to ensure that NHS trusts allocate appropriate resources and priority to research. While many NHS trust leaders have witnessed the importance of research through the COVID-19 experience, research must now be integrated in a resilient way into operational plans for system-wide change across our evolving NHS, including public health and social care alongside implementation of integrated care systems.

Even before COVID-19, the benefits of research activity in the NHS were evident. Data showed that research-active hospitals have improved outcomes for all patients and not just for participants in clinical trials. ${ }^{6,7}$ Surveys from the RCP and others have revealed a huge appetite among staff for engaging in clinical research and that the barriers of time, skills and NHS trust culture need to be addressed. ${ }^{8,9}$ At a time of concern (regarding NHS staffing and the risks of burnout), protecting time for research can help improve recruitment and retention through improved job satisfaction. ${ }^{10}$

It is therefore prescient to capitalise on this appetite for research and harness the potential of our health and care workforce as part of the NHS people plan, We are the NHS: People Plan 2020/21 - action for us all. ${ }^{11}$ Over and above the need to build capacity for NHS staff to undertake research, the decline in numbers of the clinical academic workforce per se over the last 10 years means that we must be creative, flexible and innovative in our approach to delivering research. ${ }^{12}$ All clinicians must be enabled and rewarded for research involvement.

We are humbled by the generosity of our internationally leading authors and thank them for producing a brilliant range of interesting articles that will inform, challenge and push the frontiers for research. We hope that these articles will foster important conversations among thought leaders, health leaders, political leaders and stimulate tomorrow's healthcare researchers to ensure that future healthcare is always rooted in a mindset of 'better research for better evidence for better policy for better health and wealth creation'.

In our eyes, research must 'never be the same again'!

Cheng-Hock Toh

Academic vice president, Royal College of Physicians, London, UK

Paul Stewart

Clinical vice president, The Academy of Medical Sciences, London, UK

\section{References}

1 Department of Health and Social Care. The NHS Constitution for England. DHSC, 2021. www.gov.uk/government/publications/ the-nhs-constitution-for-england/the-nhs-constitution-for-england [Accessed 08 June 2021].

2 Department of Health and Social Care, The Executive Office (Northern Ireland), Scottish Government, Welsh Government. Saving and improving lives: the future of UK clinical research delivery. GOV.UK, 2021. www.gov.uk/government/publications/thefuture-of-uk-clinical-research-delivery/saving-and-improving-livesthe-future-of-uk-clinical-research-delivery [Accessed 08 June 2021]

3 Royal College of Physicians. Delivering research for all: expectations and aspirations for the NHS in England. RCP, 2019. www. rcplondon.ac.uk/guidelines-policy/delivering-research-all-expectations-and-aspirations-nhs-england [Accessed 08 June 2021].

4 The Academy of Medical Sciences. Enhancing the NHS-academia interface. AMS, 2019. https://acmedsci.ac.uk/policy/policy-projects/ nhs-academia-interface [Accessed 08 June 2021].

5 Care Quality Commission. A new strategy for the changing world of health and social care - CQC's strategy from 2021. CQC, 2021. www. cqc.org.uk/about-us/our-strategy-plans/new-strategy-changing-worldhealth-social-care-cqcs-strategy-2021 [Accessed 08 June 2021].

6 Downing A, Morris EJ, Corrigan N et al. High hospital research participation and improved colorectal cancer survival outcomes: a population-based study. Gut 2017;66:89-96.

7 Jonker L, Fisher SJ. The correlation between National Health Service trusts' clinical trial activity and both mortality rates and Care Quality Commission ratings: a retrospective cross-sectional study. Public Health 2018;157:1-6.

8 Royal College of Physicians. Research for all? An analysis of clinical participation in research. RCP, 2020. www.rcplondon.ac.uk/projects/ outputs/research-all-analysis-clinical-participation-research [Accessed 08 June 2021].

9 Peckham S, Eida T, Zhang W et al. Creating time for research. Cancer Research UK, 2021. www.cancerresearchuk.org/sites/ default/files/creating_time_for_research_february_2021_-_supplementary_survey_report.pdf [Accessed 08 June 2021].

10 Rees MR, Bracewell M. Academic factors in medical recruitment: evidence to support improvements in medical recruitment and retention by improving the academic content in medical posts. Postgrad Med J 2019;95:323-7.

11 NHS England. We are the NHS: People Plan 2020/21 - action for us all. NHS, 2020.

12 Medical Schools Council. Survey of medical clinical academic staffing levels 2017. Medical Schools Council, 2017. http:// allcatsrgrey.org.uk/wp/download/education/medical_education/ Survey-Medical-Clinical-Academic-Staffing-Levels-2017.pdf [Accessed 8 June 2021]. 